A transfer Hamiltonian approach for an arbitrary quantum dot array in the selfconsistent field regime

S. Illera, N. Garcia-Castello, J. D. Prades, and A. Cirera

Citation: Journal of Applied Physics 112, 093701 (2012); doi: 10.1063/1.4763359

View online: https://doi.org/10.1063/1.4763359

View Table of Contents: http://aip.scitation.org/toc/jap/112/9

Published by the American Institute of Physics 


\title{
A transfer Hamiltonian approach for an arbitrary quantum dot array in the self-consistent field regime
}

\author{
S. Illera, ${ }^{\text {a) }}$ N. Garcia-Castello, J. D. Prades, and A. Cirera \\ MIND/IN2UB Departament d'Electrónica, Universitat de Barcelona, C/Martí i Franquès 1, \\ E-08028 Barcelona, Spain
}

(Received 23 May 2012; accepted 3 October 2012; published online 1 November 2012)

\begin{abstract}
A transport methodology to study electron transport between quantum dot arrays based on the transfer Hamiltonian approach is presented. The interactions between the quantum dots and between the quantum dots and the electrodes are introduced via transition rates and capacitive couplings. The effects of the local potential are computed within the self-consistent field regime. The model has been developed and expressed in a matrix form in order to make it extendable to larger systems. Transport through several quantum dot configurations has been studied in order to validate the model. Despite the simplicity of the model, well-known effects are satisfactorily reproduced and explained. The results qualitatively agree with other results obtained using more complex theoretical approaches. (C) 2012 American Institute of Physics. [http://dx.doi.org/10.1063/1.4763359]
\end{abstract}

\section{INTRODUCTION}

Confined structures have been available to the experimentalists for a very long time. The metal-oxide-semiconductor (MOS) transistor is the archetypal confined two-dimensional system. ${ }^{1}$ Nevertheless, the possibility of enhancing this confinement by embedding low-dimensional structures in an insulating matrix has caused renewed interest. These structures (quantum dots, wires, or layers) can be used in single-electron devices, ${ }^{2}$ new memory concepts, ${ }^{3}$ and photon or electroluminescent devices. ${ }^{4}$

Quantum dots (Qds) are particularly attractive because they possess discrete energy levels and quantum properties similar to natural atoms or molecules. From a theoretical point of view, research has mostly concentrated on single Qds. These simple systems have been studied using manybody approaches, including the non-equilibrium Green's function formalism (NEGFF). ${ }^{5,6}$ From a practical point of view, many novel phenomena have been discovered, such as the staircase-like current-voltage (I-V) characteristic, ${ }^{7} \mathrm{Cou}-$ lomb blockade oscillation, ${ }^{8}$ negative differential capacitance, ${ }^{9}$ and the Kondo effect in Qds. ${ }^{10}$

Researchers have recently paid much attention to electron transport through several Qds since multiple Qds provide more Feynman paths for electron transmission. ${ }^{11}$ However, up to now the only computation of transport in an extended arbitrary array of Qds has been done by Carreras et $a l .{ }^{12}$ which did not include any local potential due to selfcharge. Sun et al. ${ }^{13}$ have also studied electron transport using NEGFF for different arrangements of Qds, from one to three Qds, without including the potential due to self-charge. The inclusion of the self-charge potential using such a complex framework is usually impossible for large systems.

In this work, we use non-coherent rate equations $(\mathrm{NCRE})^{14,15}$ to study electrical transport in Qds in an extendable, arbitrary matrix of Qds, taking into account self-charge

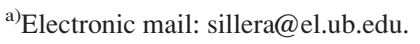

effects. In previous work, ${ }^{16}$ we applied NCRE to obtain analytical solutions for electron transport in simple cases. Using this approach each Qd is treated as a separate system; therefore, we can write a NCRE for each dot since the equations describe the relationship between the charge inside the Qds and the applied bias voltage. The interactions between the Qds and between the Qds and the electrodes are introduced by transition rates and capacitive couplings. Electron transport and charge densities inside the Qds depend on the tunnel transparency of the barriers limiting each dot. In order to effectively solve the multielectron problem, the effects of the local potential are computed within the self-consistent field (SCF) regime. Moreover, we show how our approach can easily be extended to an arbitrary number of Qds and any configuration using a matrix formalism. Therefore, this methodology allows us to simulate realistic devices based on large scale Qd arrays. Finally, we compare this methodology with NEGFF and show that both yield similar results.

\section{THEORETICAL BACKGROUND}

Our system consists of two electrodes (L lead and R lead) coupled to a central transport region. The central region contains several Qds, $N$ Qds, distributed inside an insulator matrix. In order to find the current-voltage (I-V) curve of the total system, we use the transfer Hamiltonian formalism. ${ }^{17,18}$ Using this formalism we can write an expression for the current flowing between two parts of the system. Assuming no inelastic scattering and symmetry in the transmission coefficient, ${ }^{19}$ the net current flux between two parts of the system is

$$
I_{i j}=\frac{4 \pi q}{\hbar} \int T_{i j}(E) \rho_{i}(E) \rho_{j}(E)\left(f_{j}(E)-f_{i}(E)\right) d E,
$$

where $T_{i j}(E)$ is the transmission probability, $\rho_{i}(E)$ and $\rho_{j}(E)$ are the density of states while $f_{i}(E)$ and $f_{j}(E)$ are the distribution functions (DFs) of the different parts of the system. In equilibrium, the electrochemical potential is the same throughout the whole system, and the particular DFs are described by 
the equilibrium Fermi Dirac DF. Therefore, the current between any two parts of the system is zero. If an external bias voltage (V) is applied, which drives the system out of equilibrium, the electrochemical potential of the leads will change by $\mu_{L}-\mu_{R}=q V$. From the definition of the total charge $N_{i}$ inside the $i$ th $\mathrm{Qd}$, we can write

$$
N_{i}=\int \rho_{i}(E) n_{i}(E) d E,
$$

where $n_{i}(E)$ is an unknown DF and $\rho_{i}$ is the density of states (DOS) of the $i$ th Qd. For the sake of clarity, we only consider a single state with energy level $\epsilon$ in each Qd. In order to take into account the coupling with the surrounding elements, we assign a Lorentzian shape $\operatorname{DOS}^{20}$ centered on $\epsilon$ and the halfwidth value is proportional to the strength of the coupling. We can write the evolution of the charge over time for each Qd as $N_{i}=\sum_{j} \int I_{j i} d t$, where the subscript $i$ refers to the $i$ th Qd and $j$ runs over the other components of the system.
Thus, a set of integro-differential equations are obtained for the charge evolution

$$
\begin{aligned}
\frac{d N_{i}}{d t}= & \frac{4 \pi q}{\hbar}\left(\int T_{L i} \rho_{L} \rho_{i}\left(f_{L}-n_{i}\right) d E+\int T_{R i} \rho_{R} \rho_{i}\left(f_{R}-n_{i}\right) d E\right. \\
& \left.+\sum_{j \neq i}^{(N-1)} \int T_{j i} \rho_{j} \rho_{i}\left(n_{j}-n_{i}\right) d E\right) \quad \forall i=1 \ldots N,
\end{aligned}
$$

where we explicitly write out all the current terms: the current contributions from the leads (first and second term) and the neighbor contribution (the last term). We assume that the DFs in the electrodes $\left(f_{L}\right.$ and $\left.f_{R}\right)$ are similar to the Fermi Dirac DF using different electrochemical potentials $\left(\mu_{L}\right.$ and $\left.\mu_{R}\right)$. Equation (3) can be rewritten for the steady state, and assuming no inelastic scattering we can obtain the DF in each Qd and for each energy step as a solution of the system of equations

$$
\left(\begin{array}{ccc}
-T_{L 1} \rho_{L}-T_{R 1} \rho_{R}-\sum_{j \neq 1}^{(N-1)} T_{1 j} \rho_{j} & \ldots & T_{1 N} \rho_{N} \\
\vdots & \ddots & \vdots \\
T_{1 N} \rho_{1} & \ldots & -T_{L N} \rho_{L}-T_{R N} \rho_{R}-\sum_{j \neq N}^{(N-1)} T_{N j} \rho_{j}
\end{array}\right)\left(\begin{array}{c}
n_{1} \\
\vdots \\
n_{N}
\end{array}\right)=\left(\begin{array}{c}
-T_{L 1} \rho_{L} f_{L}-T_{R 1} \rho_{R} f_{R} \\
\vdots \\
-T_{L N} \rho_{L} f_{L}-T_{R N} \rho_{R} f_{R}
\end{array}\right)
$$

The effect on the electrostatic potential inside each Qd of the voltage applied to the external electrodes must also be taken into account. The classical solution for the potential in each Qd $\left(V_{i}\right)$ involves the Poisson equation

$$
\vec{\nabla} \cdot\left(\varepsilon_{r} \vec{\nabla} V_{i}\right)=-\frac{q \Delta N_{i}}{\Omega \varepsilon_{0}},
$$

where $\varepsilon_{r}$ is the relative permittivity of the dielectric medium, $\varepsilon_{0}$ is the vacuum permittivity, and $\Omega$ is the $\mathrm{Qd}$ volume. The general solution for the potential energy $U_{i}=-q V_{i}$ in the $i$ th $\mathrm{Qd}$ is ${ }^{21}$

$$
U_{i}=\sum_{j \neq i} \frac{C_{i j}}{C_{t o t, i}}\left(-q V_{j}\right)+\frac{q^{2}}{C_{t o t, i}} \Delta N_{i}
$$

where the subscript $j$ runs over all the components of the system, $C_{i j}$ is the capacitive coupling between the different components, and $C_{t o t, i}=\sum_{j, j \neq i} C_{i j}$ is the total capacitive coupling of the $i$ th $\mathrm{Qd}$. The charge energy constant $U_{0 i}=$ $q^{2} / C_{t o t, i}$ is the increase in potential as a consequence of the injection of one electron into the $\mathrm{Qd}$ and $\Delta N_{i}$ is the change in the number of electrons, calculated with respect to the number of electrons $N_{0}$ initially in the $i$ th $\mathrm{Qd}$. The effects of local potential on each Qd, which modifies the Qd charge and the currents, should be taken into account in the Qd $\operatorname{DOS} \rho_{i}(E) \rightarrow \rho_{i}\left(E-U_{i}\right)$. From Eq. (6) we observe that the local potential depends on the increasing charge density, but at the same time the charge depends on the DOS that is modified by the local potential. These considerations impose a self-consistent solution of Eqs. (2) and (6).

\section{RESULTS AND DISCUSSION}

In this section, we first show the calculated I-V curves for different arrangements and we compare them with the results obtained using NEGFF. ${ }^{13}$ We also present the number of electrons, $N_{i}$, accumulated in the $i$ th Qd in each configuration. In these cases, analytical expressions for the current are presented as well. Finally, the extension of the model developed in Sec. II is presented as a powerful method for studying electron transport in an arbitrary extended array of Qds.

The electrochemical potentials in the two leads are set at $\mu_{L}=0$ and $\mu_{R}=-q V$. Electrons flow from the left lead to the right one. For simplicity, we consider that the transmission probability is constant and the same between all the parts of the system. We do not consider direct transmission between the leads. For clarity the DOS of the leads are considered to be constant over the whole energy range. Using this framework (transport without inelastic scattering) the position of the energy levels in the Qds plays an important role, and therefore its evolution with the applied bias voltage defines the shape of the $\mathrm{I}(\mathrm{V})$ curve. As expected, the I(V) curves exhibit strong dependence on the electrostatic coupling of the different parts of the system. We present expressions for the evolution of the energy levels with the applied bias voltage assuming that there is equal capacitive coupling between the elements which are coupled. We set a constant charge energy for all Qds: $U_{0}=0.25 \mathrm{eV}$. 


\section{A. One single Qd}

We briefly review electron transport through one Qd. Using Eq. (3) and only taking into account the lead contributions, the current can be written as

$$
I=\frac{4 \pi q}{\hbar} \int \frac{T_{R 1} T_{L 1} \rho_{L} \rho_{1} \rho_{R}}{T_{L 1} \rho_{L}+T_{R 1} \rho_{r}}\left(f_{L}-f_{R}\right) d E .
$$

Fig. 1 shows the numerical result for the current $I(V)$. In the calculation we assumed symmetric coupling with respect to the leads, $T_{R 1}=T_{L 1}=0.2{ }^{22}$ The evolution of the energy level with the applied bias voltage is

$$
\epsilon_{1}(V)=1-V / 2+U_{0} \Delta N_{1}
$$

where the second and third terms are due to the electrostatic effect. As expected, the current increases with the bias when the energy of the Qd moves across the left lead, which is $\mu_{L}=\epsilon_{1}(V) \rightarrow V \approx 2$. When $V$ is high enough, the current saturates to a constant value, as $\epsilon_{1}(V)$ is placed between the two electrochemical potentials of the leads. Fig. 1(b) shows the dependence of the electron number on the applied bias.

\section{B. Two Qds}

We now study the case of two Qds. There are four different connection geometries between the Qds and the leads. In our calculations we assume symmetric coupling with respect to the leads, $T_{R 1}=T_{L 1}=0.2$, and the Qd coupling $T_{12}=0.2$. We use the same energy level as Sun et al. ${ }^{13}$ in order to make the qualitative comparison between the two models possible.

\section{Parallel case}

The first configuration of two Qds is the case in which they are in parallel. Both the Qds are coupled to all the ele-

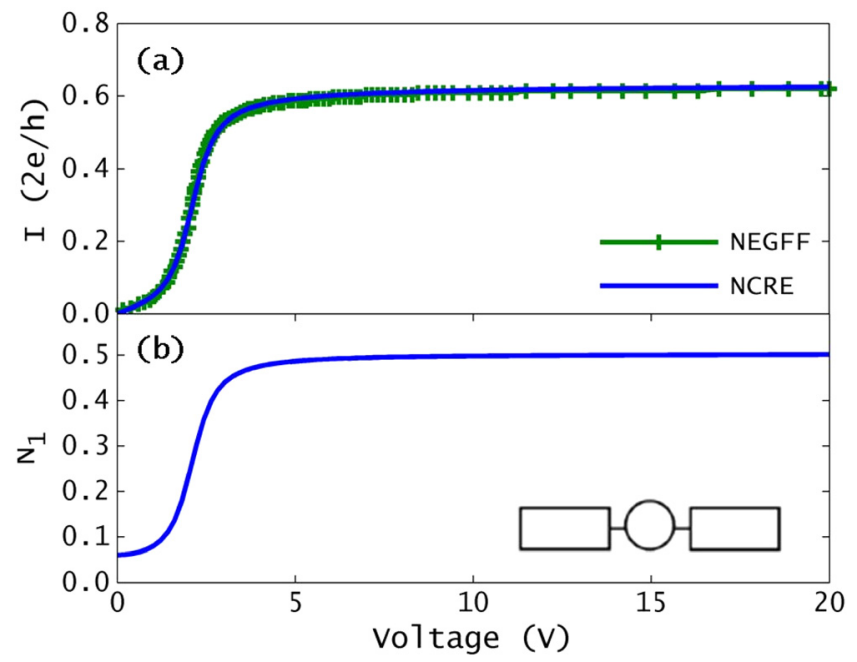

FIG. 1. (a) The I-V curve for one single Qd obtained using NCRE. We also show the NEGFF results for the same system, and the NEGFF data are taken from Sun et al. ${ }^{13}$ (b) The electron number in the Qd as a function of the applied bias V. The inset shows the connection geometry. The rectangles represent two leads and the circle represents a Qd. ments of the system: the leads and the neighboring Qd. In this configuration the expressions for the current are

$$
\begin{aligned}
I_{1}= & \frac{4 \pi q}{\hbar} \int \frac{T_{L 1} T_{R 1}\left(T_{L 1} \rho_{L}+T_{1 R} \rho_{R}+T_{12}\left(\rho_{1}+\rho_{2}\right)\right) \rho_{L} \rho_{R} \rho_{1}}{D_{2}} \\
& \times\left(f_{L}-f_{R}\right) d E, \\
I_{2}= & \frac{4 \pi q}{\hbar} \int \frac{T_{L 1} T_{R 1}\left(T_{L 1} \rho_{L}+T_{1 R} \rho_{R}+T_{12}\left(\rho_{1}+\rho_{2}\right)\right) \rho_{L} \rho_{R} \rho_{2}}{D_{2}} \\
& \times\left(f_{L}-f_{R}\right) d E,
\end{aligned}
$$

where $D_{2}=\left(T_{1 R} \rho_{L}+T_{1 L} \rho_{R}\right)^{2}+T_{1 L} T_{12} \rho_{R}\left(\rho_{1}+\rho_{2}\right)+T_{L 1} T_{12} \rho_{L}$ $\left(\rho_{1}+\rho_{2}\right)$. The energy level of each $\mathrm{Qd}$ is

$$
\begin{gathered}
\epsilon_{1}(V)=1-q V / 3-q V_{2} / 3+U_{0} \Delta N_{1}, \\
\epsilon_{2}(V)=3.5-q V / 3-q V_{1} / 3+U_{0} \Delta N_{2} .
\end{gathered}
$$

The total and partial currents are shown in Fig. 2(a). The I-V curve shows two steps when the energy levels of the Qds are placed between the electrochemical potentials of the leads. This case is equivalent to a single Qd with two energy levels. Fig. 2(b) shows the electron number $n_{i}$ with the bias voltage applied. The charge increases until it reaches the saturation value.

\section{Serial case}

The second type of arrangement is the case of two Qds in a serial configuration. The system is shown in the inset of Fig. 3(a). Each Qd only interacts with one lead and the other Qd. In this case, the expression for the current is

$I=\frac{4 \pi q}{\hbar} \int \frac{T_{L 1} T_{12} T_{2 R} \rho_{L} \rho_{1} \rho_{2} \rho_{R}}{T_{L 1} T_{12} \rho_{1} \rho_{L}+T_{L 1} T_{2 R} \rho_{R} \rho_{L}+T_{12} T_{2 R} \rho_{2} \rho_{R}}\left(f_{L}-f_{R}\right) d E$,

and the evolution of the energy level of each Qd with the applied bias voltage is

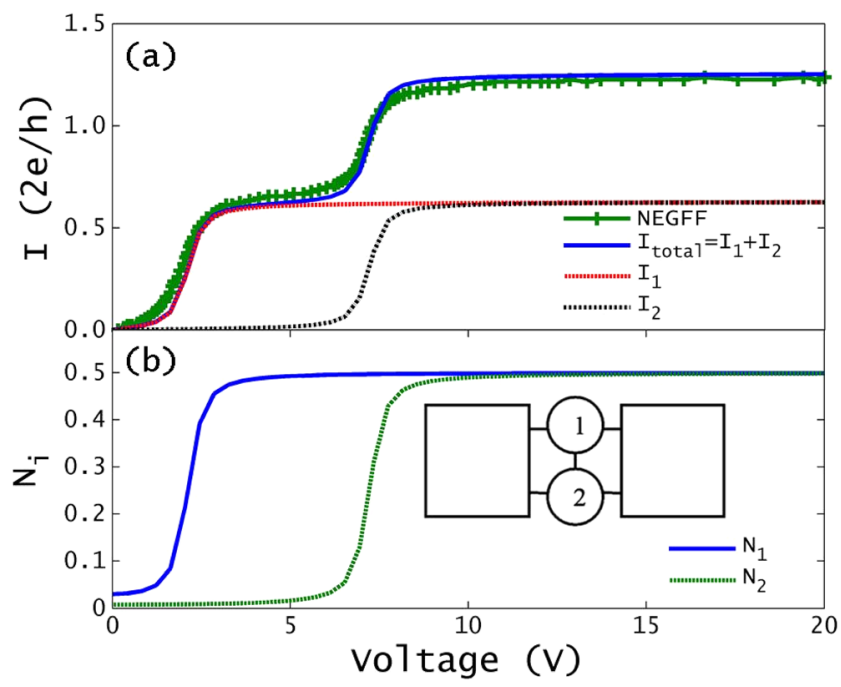

FIG. 2. (a) The total I-V curve and partial I-V curves obtained using NCRE for a parallel configuration. The NEGFF results are taken from Sun et al. ${ }^{13}$ (b) The electron number in the Qds as a function of the applied bias V. 


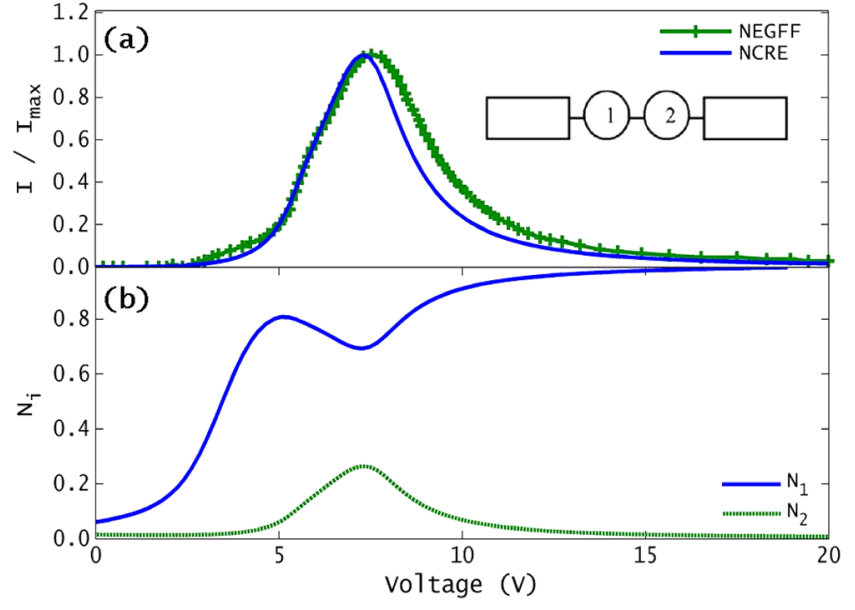

FIG. 3. (a) The I-V curve for two Qds in a serial configuration obtained using NCRE. We also show the NEGFF results for the same system, the NEGFF data are taken from Sun et al. ${ }^{13}$ The inset shows the connection geometry. (b) The electron number in the Qds as a function of the applied bias V.

$$
\begin{gathered}
\epsilon_{1}(V)=1-q V_{2} / 2+U_{0} \Delta N_{1}, \\
\epsilon_{2}(V)=3.5-q V / 2-q V_{1} / 2+U_{0} \Delta N_{2},
\end{gathered}
$$

where we have assumed that the Qds are only coupled to each other and to one lead. In order to have current flowing through the system, the energy levels must lie between the electrochemical potentials of the leads and overlapping of the Qd energy levels is also necessary. This means that the electrons need available states in each part of the system in order to move from the left lead to the right lead. When the energy levels are equal, $\epsilon_{1}=\epsilon_{2} \rightarrow V \approx 7.5$, there is maximum overlapping between Qd DOS, the current is maximum, and the system is in a resonance state; therefore, the channel is open. When the voltage increases further, the overlapping of the Qd DOS decreases. Therefore, a negative differential resistance appears. ${ }^{23}$ In Fig. 3(b) we show the evolution of the charge in each Qd $N_{i}$ as a function of the applied voltage, $\mathrm{V}$. Initially, $N_{1}$ increases since the channel between the first and second Qd is closed. At the resonant condition, the channel between the Qds opens, and some charge stored in the first Qd flows to the second Qd. At higher voltages, the channel closes again and $N_{1}$ stores all the incoming charge, while $N_{2}$ loses its charge.

\section{Other two-Qd configurations}

We first examine the case in which one Qd interacts with the two leads and is also connected to the second Qd, while the second Qd is only connected to the first Qd. The current is

$$
I=\frac{4 \pi q}{\hbar} \int \frac{T_{R 1} T_{L 1} \rho_{L} \rho_{1} \rho_{R}}{T_{L 1} \rho_{L}+T_{R 1} \rho_{r}}\left(f_{L}-f_{R}\right) d E
$$

and the energy levels are

$$
\begin{gathered}
\epsilon_{1}(V)=1-q V / 3-q V_{2} / 3+U_{0} \Delta N_{1}, \\
\epsilon_{2}(V)=3.5-q V_{1}+U_{0} \Delta N_{2} .
\end{gathered}
$$

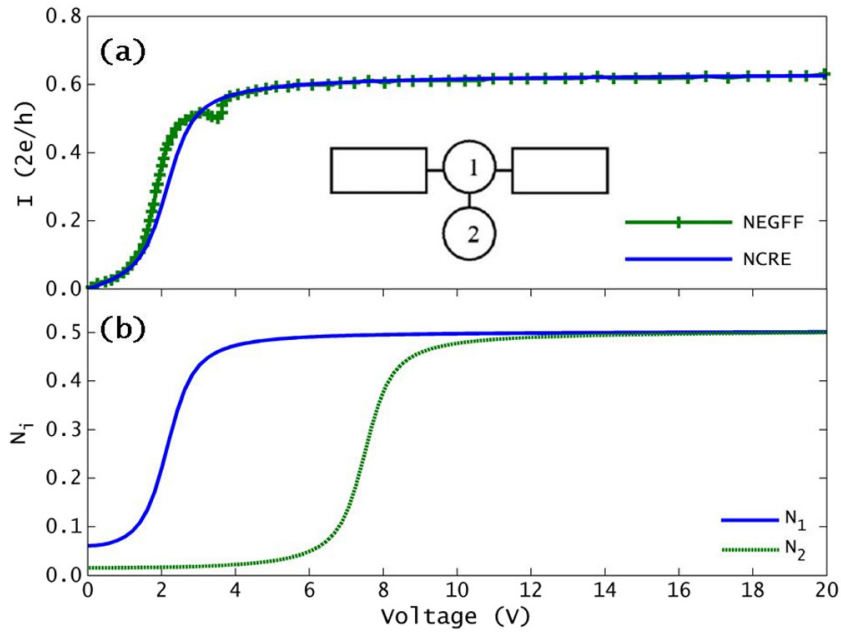

FIG. 4. (a) The I-V curve, for the configuration plotted in the inset, obtained using NCRE. We also show the NEGFF results for the same system, the NEGFF data are taken from Sun $e^{a l} .^{13}$ (b) The electron number in the Qds as a function of the applied bias $\mathrm{V}$.

The expression obtained for the current (Eq. (13)) is the same as the one we obtained for the single Qd case. The DF in the second Qd is the same as in the first Qd; therefore, the current between the Qds is zero. The results are presented in Fig. 4.

The second arrangement of Qds is shown in the inset of Fig. 5. The expressions for the current are

$$
\begin{gathered}
I_{1}=\frac{4 \pi q}{\hbar} \int \frac{T_{1 R} \rho_{1} \rho_{R}\left(T_{R 2} \rho_{R} T_{L 1} \rho_{L}+T_{12} \rho_{1} T_{L 1} \rho_{L}\right)}{D}\left(f_{L}-f_{R}\right) d E, \\
I_{2}=\frac{4 \pi q}{\hbar} \int \frac{T_{2 R} \rho_{2} \rho_{R} T_{12} \rho_{1} T_{L 1} \rho_{L}}{D}\left(f_{L}-f_{R}\right) d E
\end{gathered}
$$

where $D=T_{2 R} \rho_{R} T_{L 1} \rho_{L}+T_{R 1} T_{R 2} \rho_{R}^{2}+T_{R 2} \rho_{R} T_{12} \rho_{2}+T_{12} \rho_{1}$ $T_{L 1} \rho_{L}+T_{R 1} \rho_{R} T_{12} \rho_{1}$ and the total current is $I=I_{1}+I_{2}$. The energy level is

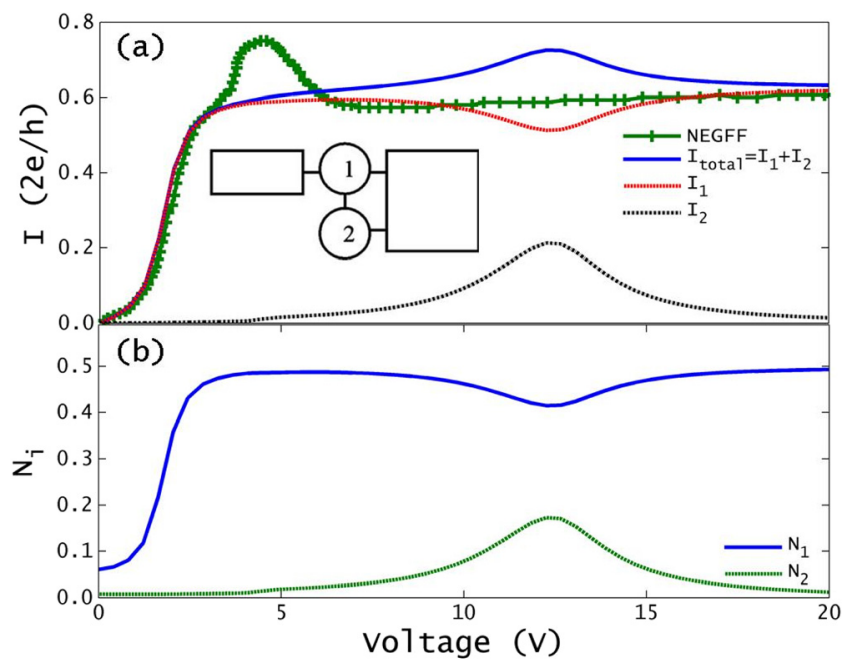

FIG. 5. (a) The total I-V curve and partial I-V curves obtained using NCRE for the configuration showed in the inset. The NEGFF results are taken from Sun et al. ${ }^{13}$ (b) The electron number in the Qds as a function of the applied bias $\mathrm{V}$. 


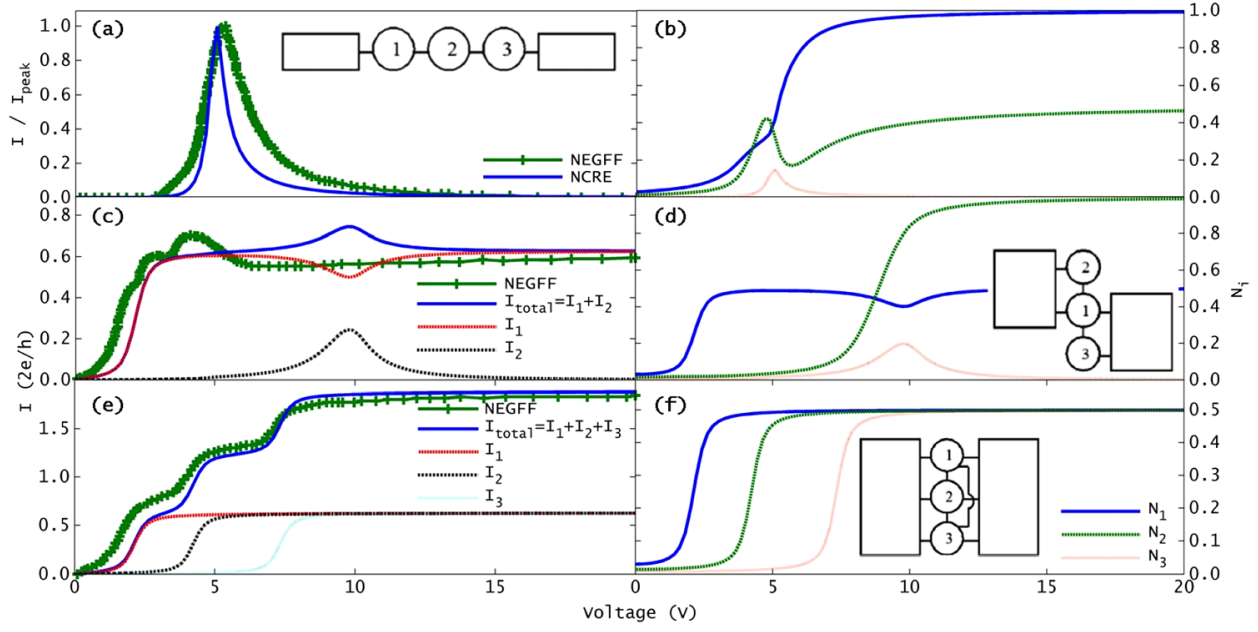

FIG. 6. (a),(c),(e) The I-V curves and the electron number (b),(d),(f), respectively, for three Qds with different configuration. The insets show the connection geometry. The NEGFF results are taken from Sun et al. $^{13}$

$$
\begin{gathered}
\epsilon_{1}(V)=1-q V / 3-q V_{2} / 3+U_{0} \Delta N_{1}, \\
\epsilon_{2}(V)=3.5-q V_{1} / 2+U_{0} \Delta N_{2} .
\end{gathered}
$$

In this case we show the total and partial currents. The I-V partial current demonstrates interesting behavior. The current through the first Qd is similar to that in the single Qd configuration, but the current through the second resembles the slope of a resonant state. This can easily be understood by taking the following into account: if the channel between the two Qds is closed, the current only flows through the first Qd. When the Qd1-Qd2 channel is opened, Qd2 also conducts. Just as in the previous case, when the voltage increases the overlapping decreases and the $\mathrm{Qd} 2$ current decreases.

\section{Three Qds}

The methodology developed in the first section can easily be extended to more complicated systems. Here, we present the results for some configurations based on three Qds. The analytical expressions for the current are too large to write out here, but in Figs. 6(a), 6(c), and 6(e) we show the I$\mathrm{V}$ curves and the charge in each Qd (Figs. 6(b), 6(d), and 6(f)). As we have shown before, the energy levels play an important role in the I-V and N-V curves; using Eq. (6), we can write each energy level as a function of the applied bias voltage

$$
\begin{gathered}
\epsilon_{1}(V)=1-\sum_{j} \frac{C_{1 j}}{C_{\text {total } 1}} V_{j}+U_{0} \Delta N_{1}, \\
\epsilon_{2}(V)=2-\sum_{j} \frac{C_{2 j}}{C_{\text {total } 2}} V_{j}+U_{0} \Delta N_{2}, \\
\epsilon_{3}(V)=3.5-\sum_{j} \frac{C_{3 j}}{C_{\text {total3 }}} V_{j}+U_{0} \Delta N_{3},
\end{gathered}
$$

where the subscript $j$ runs over all the connected elements of the system. The Qd-lead coupling and the interdot coupling are set equal to $T_{i j}=0.2$. We use the same energy level as Sun et al. ${ }^{13}$ in order to make qualitative comparison between the two models possible. In the inset of Fig. 6, we show the scheme of the system under study.

\section{Large Qd arrangements}

To conclude, we present the results for larger systems that are closely related to experimental measurements. The systems are formed of 100 Qds placed in a parallel configuration, in a serial configuration and in a geometrical array $(10 \times 10)$. The total $\mathrm{I}-\mathrm{V}$ curves and the geometries are presented in Fig. 7. The Qd-lead coupling and the interdot coupling are set equal to $T_{i j}=0.2$. The capacitance between the linked elements is also equal. In order to represent an experimental system, we consider that the value of the energy level of each dot follows a normal distribution with a mean value of $1 \mathrm{eV}$ and deviation of $0.2 \mathrm{eV}$. This represents typical experimental distributions. The relationship between the Qd radius and the energy level is a well-known effect, and it is related to the quantum confinement of the electrons. ${ }^{24}$

The I-V curves demonstrate interesting behavior. First, in the parallel case (Fig. 7(a)) the I-V curve has a staircase-

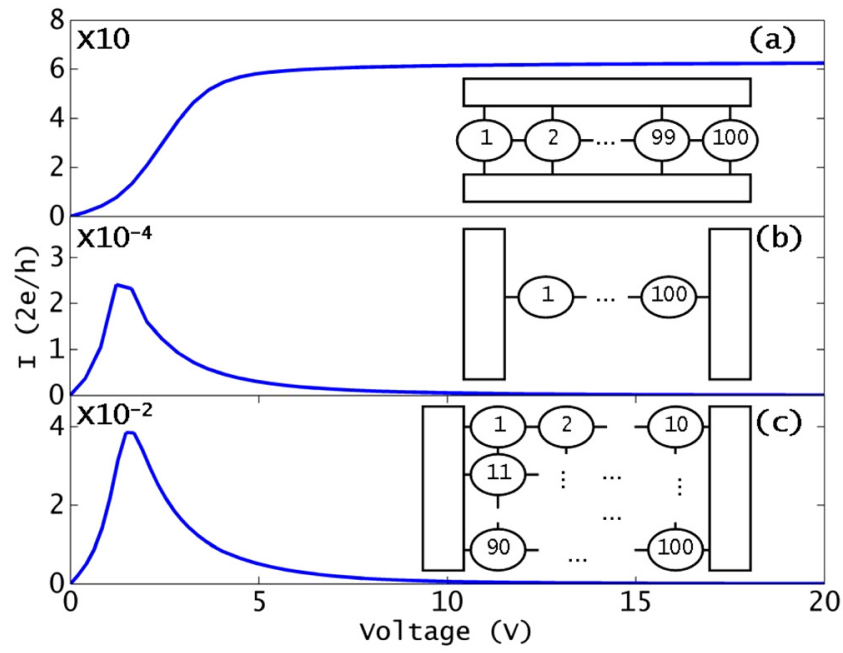

FIG. 7. The I-V curves for the larger systems: (a) 100 Qds in parallel configuration, (b) 100 Qds in serial configuration, and (c) 100 Qds in an array disposition $10 \times 10$. The energy level distribution has been generated for each system, results correspond to one single set of these normally distributed Qds and energy levels. 
like structure, and the current saturates to a constant value at high bias. As we have seen before, in the parallel configuration each Qd acts as an independent channel; therefore, the total current is the sum of all the partial currents. As expected, the saturation current is 100 times the saturation current of a single dot.

For the serial configuration (Fig. 7(b)) we obtain a current peak, as we expect, since the resonant state is necessary for there to be electron transport in this configuration. The maximum value of the peak is hard to determine because it depends on the transmission coefficient, but it also depends of the overlapping between the DOS of the Qds.

Concerning the array configuration (Fig. 7(c)), the I-V curve is determined by a combination of the two previous cases. In order for there to be transport, the resonant state condition must be fulfilled; therefore, a current peak appears, but the total current is the sum of the partial currents of each row.

\section{E. Comparison with NEGFF}

Finally, we compare the results obtained using the proposed approach to the results of Sun et al. ${ }^{13}$ In their paper, they used the NEGFF to study electron transport between one, two and three Qds in several configurations. Their I-V results are reproduced in our figures (NEGFF in the legend). The main results are:

- The results presented in Figs. 1, 2, 3, 6(a), and 6(c) are in agreement across the two approaches. For the serial configuration (Fig. 3), the differences are due to the different values of the Qd coupling; we also obtain a resonant peak when the energy levels of the Qds are in a resonant state. The resonant state is strongly dependent on the capacitive coupling of the Qds, as the energy level with the applied bias voltage depends on the capacitive coupling of the Qd.

- In the parallel configuration we obtain the same staircase shape, but, in our case we also take into account the charge terms, therefore our steps occur at higher voltages.

- The main difference appears in the case described in Fig. 4. For this configuration, Sun et al. predicts an antiresonance effect. We do not recover this effect because our model considers each Qd as a separate quantum system. For this reason, our approach is known as a non-coherent model.

- For the systems presented in Figs. 5 and 6(b) we obtain similar results. The position of the current peak is different because Sun et al. assumes that the bias is uniformly applied throughout the whole system; meanwhile, we take into account all the electrostatic coupling between the different parts of the system.

As we have shown that the electrostatic coupling plays an important role when it comes to determine the I-V characteristics of the system. The electrostatic effect has two terms: the first term is determined by the influence of the leads and the neighboring Qd, and it is described by the capacitive coupling of the Qd to its surroundings. The second term takes into account the charge stored inside the Qd, this effect is related to the electron-electron interaction, and the self-consistent solution of Eqs. (2) and (6) is the first approach to introduce many-body effects, such as the Coulomb blockade. If we create nanodevices in order to take advantage of the quantization of the current, only a small number of discrete energy levels are available for conduction; accurate control of the energy levels via the applied bias voltage is one of the most important points that we need to take into account. Therefore, good modelization of the Qd-Qd and Qd-lead capacitances is necessary.

This paper precedes future research into which realistic DOS, energy dependent transmission coefficients, as well as realistic capacitive couplings can be introduced.

\section{CONCLUSION}

We propose a theoretical model to study electron currents in systems based on Qds. This model is based on the transfer Hamiltonian approach and computes the I-V and $\mathrm{N}-\mathrm{V}$ curves in the SCF regime, using NCREs. Our approach provides a simple and transparent method for describing electron transport. Due to the simplicity of the model, it can be easily extended to analyze arbitrary large arrays of Qds that may be of interest in technological applications. Despite its simplicity and in contrast with other approaches, the effect of self-charge has been taken into account by solving the Poisson equation with appropriate boundary conditions for each Qd. As expected, the calculation of the local potential inside each Qd is one of the most critical points, since the I-V curves depend on the energy level.

In order to show the potential of this method to analyze realistic configurations, we have studied electron transport between different Qd configurations. We have also compared the NCRE results with well-established data obtained with NEGFF. The success of this comparison shows that NCRE is a powerful and intuitive method for describing electron transport.

\section{ACKNOWLEDGMENTS}

N. Garcia acknowledges the Spanish MICINN for her Ph.D. grant within the FPU program. A. Cirera acknowledges support from the ICREA academia program. The authors thankfully acknowledge the computer resources, technical expertise, and assistance provided by the Barcelona Supercomputing Center - Centro Nacional de Supercomputación. The research leading to these results has received funding from the European Community's Seventh Framework Programme (FP7/2007-2013) under Grant Agreement No. 245977.

${ }^{1}$ T. Ando, A. B. Fowler, and F. Stern, Rev. Mod. Phys. 54, 437 (1982).

${ }^{2}$ U. Meirav and E. B. Foxman, Semicond. Sci. Technol. 11, 255 (1996).

${ }^{3}$ S. Tiwari, F. Rana, K. Chan, L. Shi, and H. Hanafi, Appl. Phys. Lett. 69, 1232 (1996).

${ }^{4}$ D. J. Lockwood, Semiconductor and Semimetals, Light Emission in Silicon From Physics to Devices (Academic, San Diego, 1998).

${ }^{5}$ A. L. Yeyati, A. M. Rodero, and F. Flores, Phys. Rev. Lett. 71, 2991 (1993).

${ }^{6}$ Y. Meir, N. S. Wingreen, and P. A. Lee, Phys. Rev. Lett. 66, 3048 (1991).

${ }^{7}$ J. B. Barner and S. T. Ruggiero, Phys. Rev. Lett. 59, 807 (1987).

${ }^{8}$ J. Weis, R. J. Haug, K. v. Klitzing, and K. Ploog, Phys. Rev. Lett. 71, 4019 (1993). 
${ }^{9}$ S. D. Wang, Z. Z. Sun, N. Cue, H. Q. Xu, and X. R. Wang, Phys. Rev. B 65, 125307 (2002).

${ }^{10}$ W. G. van der Wiel, S. D. Franceschi, T. Fujisawa, J. M. Elzerman, S. Tarucha, and L. P. Kouwenhoven, Science 289, 2105 (2000).

${ }^{11}$ W. Gong, Y. Zheng, Y. Liu, and T. Lü, Physica E (Amsterdam) 40, 618 (2008).

${ }^{12}$ J. Carreras, O. Jambois, S. Lombardo, and B. Garrido, Nanotechnology 20, 155201 (2009).

${ }^{13}$ Z. Z. Sun, R. Q. Zhang, W. Fan, and X. R. Wang, J. Appl. Phys. 105, 043706 (2009).

${ }^{14}$ D. V. Averin, A. N. Korotkov, and K. K. Likharev, Phys. Rev. B 44, 6199 (1991).

${ }^{15}$ S. A. Gurvitz, Phys. Rev. B 57, 6602 (1998).
${ }^{16}$ S. Illera, J. D. Prades, A. Cirera, and A. Cornet, Europhys. Lett. 98, 17003 (2012).

${ }^{17}$ M. C. Payne, J. Phys. C: Solid State Phys. 19, 1145 (1986).

${ }^{18}$ M. Passoni and C. E. Bottani, Phys. Rev. B 76, 115404 (2007).

${ }^{19}$ D. Supriyo, Electronic Transport in Mesoscopic Systems (Cambridge University Press, 1995).

${ }^{20}$ I. P. Batra, Solid State Commun. 124, 463 (2002).

${ }^{21}$ S. Datta, Nanotechnology 15, S433 (2004).

${ }^{22}$ We use similar transmission values than Sun et al. (Ref. 13) in order to make possible the qualitative comparison between models.

${ }^{23}$ M. Borgstrom, T. Bryllert, T. Sass, B. Gustafson, L.-E. Wernersson, W. Seifert, and L. Samuelson, Appl. Phys. Lett. 78, 3232 (2001).

${ }^{24}$ J. P. Proot, C. Delerue, and G. Allan, Appl. Phys. Lett. 61, 1948 (1992). 